\title{
On Porous Matrices with Three Delay Times: A Study in Linear Thermoelasticity
}

\author{
Manuela Carini ${ }^{1, *(1)}$ and Vittorio Zampoli ${ }^{2}$ (1) \\ 1 Department of Environmental Engineering, University of Calabria, DIAm, via Pietro Bucci 42/B, \\ 87036 Arcavacata di Rende (CS), Italy \\ 2 Department of Information and Electrical Engineering and Applied Mathematics, University of Salerno, \\ DIEM, via Giovanni Paolo II, 84084 Fisciano (SA), Italy; vzampoli@unisa.it or zampoli@gmail.com \\ * Correspondence: manuela.carini@unical.it
}

Received: 7 February 2020; Accepted: 3 March 2020; Published: 7 March 2020

\begin{abstract}
Through the present work, we want to lay the foundation of the well-posedness question for a linear model of thermoelasticity here proposed, in which the presence of voids into the elastic matrix is taken into account following the Cowin-Nunziato theory, and whose thermal response obeys a three-phase lag time-differential heat transfer law. By virtue of the linearity of the model investigated, the basic initial-boundary value problem is conveniently modified into an auxiliary one; attention is paid to the uniqueness question, which is addressed through two alternative paths, i.e., the Lagrange identity and the logarithmic convexity methods, as well as to the continuous dependence issue. The results are achieved under very weak assumptions involving constitutive coefficients and delay times, at most coincident with those able to guarantee the thermodynamic consistency of the model.
\end{abstract}

Keywords: three-phase lag thermoelasticity; uniqueness; Lagrange identity; logarithmic convexity; continuous dependence

MSC: 74A15; 74F05; 74F10

\section{Introduction}

The use of multiple relaxation times or phase lags in heat conduction constitutive equations is today more than ever a subject of ever-increasing interest, especially with regard to the related time-differential formulations: this is driven by the potential of such models to accurately predict an actual physical phenomenology in reference to extremely small spatial and temporal contexts. The literature on the subject, and more in general on topics involving multi-phase lag approaches, is absolutely extensive (we report only by way of example the selection [1-22] which provides an approximate idea of the temporal extent characterizing the interest on the theme) and in particular, in the last years, the number of research works investigating the so-called three-phase lag model of heat conduction is rapidly increasing. To sum up the issue, we must remember that Roy Choudhuri [2] proposed the addition of a further phase lag associated with the temperature displacement variable to the two relaxation times previously introduced by Tzou (see [1] and the references therein) and related to the heat flux vector and temperature gradient, where the above mentioned thermal displacement variable is attributable to Green and Naghdi [23-25].

The present work has to be considered as the natural continuation of the path recently traced in [26,27], where porous material matrices were investigated when coupled with heat transfer phenomena modeled through time-differential constitutive laws with two relaxation times. This was done not only in terms of well-posedness of the theory, but also obtaining interesting information 
about the influence of the voids on the model's response to certain external stimulations. In the present case, assuming that for adequately short spatial scales the distortions due to thermal effects are small enough to allow to be described under linear hypotheses, we investigate a thermoelastic porous medium following the Cowin-Nunziato model (see [28-31]), but this time adding a third relaxation time according to [2].

The issue appears particularly relevant also in light of the unexpected indications obtained through the numerical simulations in [27] and, in our opinion, it can actively contribute to the comprehension of the thermal exchange mechanisms involving materials with a porous skeleton at micro- and nanoscales in very fast transients. As for the hypothesis of thermal effects small enough to allow the activation of linearity assumptions, we believe that it is consistent with the evolution of a phenomenon that occurs in an extremely rapid transient; coherently, any involvement of thermal deformations of greater magnitude would probably entail a real behavior of the material gradually less superimposable to that predicted by the current model. The attention paid to the knowledge of mechanical and thermal properties of porous materials is still very high (see e.g., [32,33]). Anyway, for a more detailed discussion about the possible applications of elastic and thermoelastic media with voids and the related growing interest in physical properties of such materials, we refer the reader to the Introduction of [26]. Going into the detail of the model studied, we remember that in [34] the authors prove uniqueness and continuous dependence of the solution for a linear thermoelastic model without voids under three-phase lag hypotheses while, substantially in parallel and in addition to other results, they define in [35] the restrictions under which its thermodynamic consistency is guaranteed.

Summarizing, our aim is to give a contribution to the study of the well-posedness of a linear thermoelastic model for which the presence of pores into the elastic matrix is taken into account according to the Cowin and Nunziato theory, and whose thermodynamic response obeys a three-phase lag time-differential law. In particular, uniqueness and continuous dependence of the solution are demonstrated proceeding through the following scheme: in Section 2, the basic equations of the time-differential three-phase lag model for a porous material are described along with the presentation of related mathematical manipulations; suitable initial-boundary value problems are also defined. In Section 3, the uniqueness of the solution is proved through the Lagrange identity method, while in Section 4 a continuous dependence result is proposed with respect to the external data. In Section 5 a further and alternative uniqueness result is given, this time via the logarithmic convexity method. Finally, some concluding remarks are given. We emphasize that in [26] something similar has been done taking into account only two relaxation times; differently, in this case, two different approaches aimed at verifying the uniqueness of the solution are proposed besides the proof of a continuous dependence theorem.

\section{Mathematical Formulation: Identification of the Proposed Thermoelastic Model}

We premise that our reference will be the work of Ieşan [30] in order to take into account the presence of pores into the thermoelastic matrix (similarly to [26,27]). Our study is framed into a fixed Cartesian system of axes $O x_{1} x_{2} x_{3}$, and the common summation and differentiation conventions are used. In particular the sum is implied in case of repeated subscripts, which range from 1 to 3 , as well as a comma followed by a subscript denotes differentiation with respect to the spatial coordinate; by extension, one or more overlying dots stand for time differentiation. As an alternative in case of vector notation, bold letters may also be used. We imagine that a regular enough portion $B$ of the three-dimensional space is occupied by an anisotropic inhomogeneous linear material with pores in the field of thermoelasticity, whose nature is modeled under the Cowin and Nunziato theory [28] and following Ieşan [29-31], i.e., the volume fraction field corresponding to the pores is taken as an independent kinematical variable. Moreover, the thermal behavior is affected by the presence of three distinct relaxation times; all the functions involved are considered sufficiently regular for our purposes. Therefore, the model is the following. 
The motion equations are:

$$
t_{j i, j}+\rho f_{i}=\rho \ddot{u}_{i}, \quad \text { in } B \times(0, \infty) .
$$

Here the stress tensor has components $t_{i j}$, while $\rho$ indicates the mass density of the medium. Moreover, in terms of components, the vector denoting the external body force per unit mass is $f_{i}$, and the notation $u_{i}$ is attributed to the displacement vector.

The balance of equilibrated forces is:

$$
g=\rho \chi \ddot{\varphi}-h_{i, i}-\rho l, \quad \text { in } B \times(0, \infty) .
$$

Here, $g$ is the (intrinsic) equilibrated body force (as in [29], $g$ does not depend on $\partial \varphi / \partial t$ ), $\chi$ is the equilibrated inertia, $\varphi=v-v_{0}$ where $v$ is the volume distribution function and $v_{0}$ is $v$ in the reference configuration. In addition, the components of the equilibrated stress vector are $h_{i}$, while $l$ represents the (extrinsic) equilibrated body force per unit mass.

The energy equation, where $T_{0}$ is the constant and strictly positive ambient temperature, is:

$$
\rho T_{0} \dot{\eta}=q_{i, i}+\rho s, \quad \text { in } B \times(0, \infty) .
$$

Here, $\eta$ is the unit mass entropy and $q_{i}$ are the components of the heat flux vector. Moreover, $s$ is the external rate of heat supply per unit mass.

The constitutive equations are:

$$
\begin{aligned}
& t_{i j}=C_{i j k l} e_{k l}+B_{i j} \varphi+D_{i j k} \varphi_{, k}-M_{i j} \dot{\alpha}, \\
& h_{i}=A_{i j} \varphi_{, j}+D_{r s i} e_{r s}+d_{i} \varphi-a_{i} \dot{\alpha}, \\
& g=-B_{i j} e_{i j}-\xi \varphi-d_{i} \varphi_{, i}+m \dot{\alpha}, \\
& \rho \eta=M_{i j} e_{i j}+a \dot{\alpha}+m \varphi+a_{i} \varphi_{, i}, \\
& q_{i}+\tau_{q} \dot{q}_{i}+\tau_{q}^{2} \ddot{q}_{i} / 2=K_{i j} \beta_{j}+\left(k_{i j}+\tau_{\alpha} K_{i j}\right) \dot{\beta}_{j}+\tau_{T} k_{i j} \ddot{\beta}_{j}, \quad \text { in } \bar{B} \times[0, \infty) .
\end{aligned}
$$

Here, $\alpha$ is the thermal displacement variable and $T$, the temperature variation referred to $T_{0}$, is equal to $\dot{\alpha}$. Moreover, again in terms of components, $e_{i j}$ is the strain tensor and $\beta_{i}$ is the thermal displacement gradient, defined through the following geometrical relations:

$$
e_{i j}=\left(u_{i, j}+u_{j, i}\right) / 2, \quad \beta_{j}=\alpha_{, j}, \quad \text { in } \bar{B} \times[0, \infty) .
$$

Again, $C_{i j k l}, B_{i j}, D_{i j k}, M_{i j}, A_{i j}, d_{i}, a_{i}, \xi, m$ and $a$, as well as $k_{i j}$ and $K_{i j}$ are tensors depending only on the variables $x_{k}$, and for them the symmetries listed below are assumed to be satisfied:

$$
C_{i j k l}=C_{k l i j}=C_{j i k l}, \quad B_{i j}=B_{j i}, \quad D_{i j k}=D_{j i k}, \quad M_{i j}=M_{j i}, \quad A_{i j}=A_{j i}, \quad k_{i j}=k_{j i}, \quad K_{i j}=K_{j i} .
$$

The relaxation times $\tau_{q}, \tau_{\alpha}$ and $\tau_{T}$ are taken strictly positive. The initial conditions are selected as follows:

$$
\begin{aligned}
& u_{i}(\mathbf{x}, 0)=u_{i}^{0}, \quad \dot{u}_{i}(\mathbf{x}, 0)=\dot{u}_{i}^{0}, \quad \varphi(\mathbf{x}, 0)=\varphi^{0}, \quad \dot{\varphi}(\mathbf{x}, 0)=\dot{\varphi}^{0}, \quad \alpha(\mathbf{x}, 0)=0, \\
& \dot{\alpha}(\mathbf{x}, 0)=T^{0}, \quad q_{i}(\mathbf{x}, 0)=q_{i}^{0}, \quad \dot{q}_{i}(\mathbf{x}, 0)=\dot{q}_{i}^{0}, \quad \mathbf{x} \in \bar{B},
\end{aligned}
$$

where $u_{i}^{0}(\mathbf{x}), \dot{u}_{i}^{0}(\mathbf{x}), \varphi^{0}(\mathbf{x}), \dot{\varphi}^{0}(\mathbf{x}), T^{0}(\mathbf{x}), q_{i}^{0}(\mathbf{x})$ and $\dot{q}_{i}^{0}(\mathbf{x})$ must be understood as prescribed functions on $\bar{B}$. The choice of $\alpha(\mathbf{x}, 0)=0$ is natural since, by definition

$$
\alpha(\mathbf{x}, t)=\int_{0}^{t} T(\mathbf{x}, s) d s
$$


Being $n_{i}$ the unit outward normal vector to $\partial B$ and referring to the components of the surface traction vector, to the equilibrated stress and to the heat flux at a regular point of $\partial B$, respectively, as $t_{i}=t_{j i} n_{j}, h=h_{i} n_{i}$ and $q=q_{i} n_{i}$, we select the following boundary conditions:

$$
\begin{array}{lllll}
u_{i}(\mathbf{x}, t)=\tilde{u}_{i} & \text { on } \quad \bar{\Sigma}_{1} \times[0, \infty), & t_{j i}(\mathbf{x}, t) n_{j}=\tilde{t}_{i} & \text { on } & \Sigma_{2} \times[0, \infty), \\
\varphi(\mathbf{x}, t)=\tilde{\varphi} \quad \text { on } \quad \bar{\Sigma}_{3} \times[0, \infty), & h_{i}(\mathbf{x}, t) n_{i}=\tilde{h} \quad \text { on } & \Sigma_{4} \times[0, \infty), \\
\alpha(\mathbf{x}, t)=\tilde{\alpha} \quad \text { on } \quad \bar{\Sigma}_{5} \times[0, \infty), & q_{i}(\mathbf{x}, t) n_{i}=\tilde{q} \quad \text { on } \quad \Sigma_{6} \times[0, \infty) .
\end{array}
$$

Here, $\tilde{u}_{i}(\mathbf{x}, t), \tilde{t}_{i}(\mathbf{x}, t), \tilde{\varphi}(\mathbf{x}, t), \tilde{h}(\mathbf{x}, t), \tilde{\alpha}(\mathbf{x}, t)$ and $\tilde{q}(\mathbf{x}, t)$ represent once again prescribed functions and $\bar{\Sigma}_{1} \cup \Sigma_{2}=\bar{\Sigma}_{3} \cup \Sigma_{4}=\bar{\Sigma}_{5} \cup \Sigma_{6}=\partial B$. Moreover $\Sigma_{1} \cap \Sigma_{2}=\Sigma_{3} \cap \Sigma_{4}=\Sigma_{5} \cap \Sigma_{6}=\varnothing$.

The problem $\mathscr{P}$ can be identified through the Equations (1)-(5) and the initial-boundary conditions (7) and (9); the array of given data is thus $\mathscr{D}=\left\{f_{i}, l, s ; u_{i}^{0}, \dot{u}_{i}^{0}, \varphi^{0}, \dot{\varphi}^{0}, T^{0}, q_{i}^{0}, \dot{q}_{i}^{0} ; \tilde{u}_{i}, \tilde{t}_{i}, \tilde{\varphi}, \tilde{h}, \tilde{\alpha}, \tilde{q}\right\}$. The existence of a solution for the problem $\mathscr{P}$ corresponding to $\mathscr{D}$, i.e., the existence of an array $\mathscr{S}=\left\{u_{i}, \varphi, \alpha, e_{i j}, \beta_{j}, t_{i j}, h_{i}, g, \eta, q_{i}\right\}$ defined on $\bar{B} \times[0, \infty)$, will be assumed by hypothesis. Since we are going to use the linearity of the model, we also define a corresponding initial boundary value problem $\mathscr{P}_{0}$ associated with a null array of given data $\mathscr{D}$.

Similarly-for instance-to [34], we will refer to modified versions of the initial boundary value problems $\mathscr{P}$ and $\mathscr{P}_{0}$, denoting them by $\mathscr{P}^{*}$ and $\mathscr{P}_{0}^{*}$, respectively. In order to do this, we will use the compact notation:

$$
\bar{f}(t)=\int_{0}^{t} f(z) d z, \quad \overline{\bar{f}}(t)=\int_{0}^{t} \int_{0}^{s} f(z) d z d s, \quad \ldots,
$$

for any continuous function $f$ of time variable $t$; although the symbology is the same, no ambiguity will arise between such a notation and the closure of $B$. Consequently, it is possible to define the following operator:

$$
f^{*}(t)=\overline{\bar{f}}(t)+\tau_{q} \bar{f}(t)+\tau_{q}^{2} f(t) / 2
$$

and note that

$$
f^{*}(0)=\tau_{q}^{2} f(0) / 2, \quad\left(d f^{*} / d t\right)(0)=\tau_{q} f(0)+\tau_{q}^{2}(d f / d t)(0) / 2 .
$$

We also explicitly invoke the contents of the Lemmas 1 to 3 of [34] (p. 377), to which the reader can refer. In particular, the initial boundary value problem $\mathscr{P}^{*}$ consists of the following basic equations:

$$
\begin{aligned}
& t_{j i, j}^{*}+F_{i}=\rho \partial^{2} u_{i}^{*} / \partial t^{2}, \quad \text { in } B \times(0, \infty), \\
& g^{*}=\rho \chi \partial^{2} \varphi^{*} / \partial t^{2}-h_{i, i}^{*}-L, \quad \text { in } B \times(0, \infty), \\
& \rho T_{0} \partial \eta^{*} / \partial t=q_{i, i}^{*}+S, \quad \text { in } B \times(0, \infty), \quad \text { where } \\
& F_{i}(\mathbf{x}, t)=\rho f_{i}^{*}+\rho\left[u_{i}^{0}+\left(t+\tau_{q}\right) \dot{u}_{i}^{0}\right], \\
& L(\mathbf{x}, t)=\rho l^{*}+\rho \chi\left[\varphi^{0}+\left(t+\tau_{q}\right) \dot{\varphi}^{0}\right], \\
& S(\mathbf{x}, t)=\rho s^{*}+T_{0}\left(t+\tau_{q}\right)\left(M_{i j} u_{i, j}^{0}+a T^{0}+m \varphi^{0}+a_{i} \varphi_{, i}^{0}\right)
\end{aligned}
$$


and

$$
\begin{aligned}
& t_{i j}^{*}=C_{i j k l} l_{k l}^{*}+B_{i j} \varphi^{*}+D_{i j k} \varphi_{, k}^{*}-M_{i j} \partial \alpha^{*} / \partial t, \quad \text { in } \bar{B} \times[0, \infty), \\
& h_{i}^{*}=A_{i j} \varphi_{, j}^{*}+D_{r s i} e_{r s}^{*}+d_{i} \varphi^{*}-a_{i} \partial \alpha^{*} / \partial t, \quad \text { in } \bar{B} \times[0, \infty), \\
& g^{*}=-B_{i j} e_{i j}^{*}-\xi \varphi^{*}-d_{i} \varphi_{, i}^{*}+m \partial \alpha^{*} / \partial t, \quad \text { in } \bar{B} \times[0, \infty), \\
& \rho \eta^{*}=M_{i j} e_{i j}^{*}+a \partial \alpha^{*} / \partial t+m \varphi^{*}+a_{i} \varphi_{, i^{\prime}}^{*} \quad \text { in } \bar{B} \times[0, \infty), \\
& q_{i}^{*}=K_{i j} \overline{\bar{\beta}}_{j}+\left(k_{i j}+\tau_{\alpha} K_{i j}\right) \bar{\beta}_{j}+\tau_{T} k_{i j} \beta_{j}+\Omega_{i}, \quad \text { in } \bar{B} \times[0, \infty), \quad \text { where } \\
& \Omega_{i}(\mathbf{x}, t)=\tau_{q}^{2} q_{i}^{0} / 2+t\left(\tau_{q} q_{i}^{0}+\tau_{q}^{2} \dot{q}_{i}^{0} / 2-\tau_{T} k_{i j} T_{, j}^{0}\right)
\end{aligned}
$$

and

$$
e_{i j}^{*}=\left(u_{i, j}^{*}+u_{j, i}^{*}\right) / 2, \quad \beta_{i}^{*}=\alpha_{, i^{\prime}}^{*} \quad \text { in } \bar{B} \times[0, \infty) ;
$$

the initial-boundary conditions (7), (9) change as follows:

$$
\begin{aligned}
& u_{i}^{*}(\mathbf{x}, 0)=\tau_{q}^{2} u_{i}^{0} / 2, \quad\left(\partial u_{i}^{*} / \partial t\right)(\mathbf{x}, 0)=\tau_{q} u_{i}^{0}+\tau_{q}^{2} \dot{u}_{i}^{0} / 2, \quad \varphi^{*}(\mathbf{x}, 0)=\tau_{q}^{2} \varphi^{0} / 2, \\
& \left(\partial \varphi^{*} / \partial t\right)(\mathbf{x}, 0)=\tau_{q} \varphi^{0}+\tau_{q}^{2} \dot{\varphi}^{0} / 2, \quad \alpha^{*}(\mathbf{x}, 0)=0, \quad\left(\partial \alpha^{*} / \partial t\right)(\mathbf{x}, 0)=\tau_{q}^{2} T^{0} / 2, \\
& q_{i}^{*}(\mathbf{x}, 0)=\tau_{q}^{2} q_{i}^{0} / 2, \quad\left(\partial q_{i}^{*} / \partial t\right)(\mathbf{x}, 0)=\tau_{q} q_{i}^{0}+\tau_{q}^{2} \dot{q}_{i}^{0} / 2, \quad \text { on } \bar{B}
\end{aligned}
$$

and

$$
\begin{array}{llll}
u_{i}^{*}(\mathbf{x}, t)=\tilde{u}_{i}^{*} & \text { on } \bar{\Sigma}_{1} \times[0, \infty), & t_{j i}^{*}(\mathbf{x}, t) n_{j}\left(=t_{i}^{*}\right)=\tilde{t}_{i}^{*} & \text { on } \Sigma_{2} \times[0, \infty), \\
\varphi^{*}(\mathbf{x}, t)=\tilde{\varphi}^{*} & \text { on } \bar{\Sigma}_{3} \times[0, \infty), & h_{i}^{*}(\mathbf{x}, t) n_{i}\left(=h^{*}\right)=\tilde{h}^{*} & \text { on } \Sigma_{4} \times[0, \infty), \\
\alpha^{*}(\mathbf{x}, t)=\tilde{\alpha}^{*} & \text { on } \bar{\Sigma}_{5} \times[0, \infty), & q_{i}^{*}(\mathbf{x}, t) n_{i}\left(=q^{*}\right)=\tilde{q}^{*} & \text { on } \Sigma_{6} \times[0, \infty) .
\end{array}
$$

Correspondingly, $\mathscr{S}^{*}=\left\{u_{i}^{*}, \varphi^{*}, \alpha^{*}, e_{i j}^{*}, \beta_{j}^{*}, t_{i j}^{*}, h_{i}^{*}, g^{*}, \eta^{*}, q_{i}^{*}\right\}$ is structured like a solution of the initial-boundary value problem $\mathscr{P}^{*}$.

\section{First Result: Uniqueness Through the Application of the Lagrange Identity Technique}

The uniqueness question regarding the initial boundary value problem $\mathscr{P}$ will be treated leaning on the modified problem $\mathscr{P}^{*}$. In detail, in view of the linearity of the model, we will prove that the problem $\mathscr{P}_{0}^{*}$ associated with null given data admits only the trivial solution $\mathscr{S}_{0}^{*}$. The development of this section will be quite synthetic, considering that the mathematical structure is superimposable to [34] (pp. 382-386), to which we refer.

Theorem 1. Let $\rho>0, \chi>0, \tau_{T}>0$ and $\tau_{\alpha} \geq \tau_{q}>0$. Moreover, suppose that $k_{i j}$ is a positive definite tensor, $K_{i j}$ is a positive semi-definite tensor, and meas $\Sigma_{5} \neq 0$ or even $a>0$. Then the problem $\mathscr{P}^{*}$ (and so $\mathscr{P}$ ) admits at most one solution.

Proof of Theorem 1. For synthesis needs, the dot notation for the time derivative can be used here without ambiguity, since trivial initial data are considered. In addition, throughout this section, null assigned data will be taken: this will not be repeated but will remain implied when necessary. Finally, the symmetry conditions (6) will be valid. We start from the following Lagrange identities, valid $\forall t>0$ and $s \in(0, t)$. The first is:

$$
\rho \frac{\partial}{\partial s}\left[u_{i}^{*}(t+s) \dot{u}_{i}^{*}(t-s)+u_{i}^{*}(t-s) \dot{u}_{i}^{*}(t+s)\right]=\rho\left[\ddot{u}_{i}^{*}(t+s) u_{i}^{*}(t-s)-\ddot{u}_{i}^{*}(t-s) u_{i}^{*}(t+s)\right] .
$$


The second is:

$$
\rho \chi \frac{\partial}{\partial s}\left[\varphi^{*}(t+s) \dot{\varphi}^{*}(t-s)+\varphi^{*}(t-s) \dot{\varphi}^{*}(t+s)\right]=\rho \chi\left[\ddot{\varphi}^{*}(t+s) \varphi^{*}(t-s)-\ddot{\varphi}^{*}(t-s) \varphi^{*}(t+s)\right] .
$$

Integrated in time and space, they lead to

$$
\begin{aligned}
& 2 \int_{B}\left[\rho u_{i}^{*}(t) \dot{u}_{i}^{*}(t)+\rho \chi \varphi^{*}(t) \dot{\varphi}^{*}(t)\right] d v=\int_{0}^{t} \int_{B}\left\{\rho\left[\ddot{u}_{i}^{*}(t-s) u_{i}^{*}(t+s)-\ddot{u}_{i}^{*}(t+s) u_{i}^{*}(t-s)\right]\right. \\
& \left.+\rho \chi\left[\ddot{\varphi}^{*}(t-s) \varphi^{*}(t+s)-\ddot{\varphi}^{*}(t+s) \varphi^{*}(t-s)\right]\right\} d v d s .
\end{aligned}
$$

Using Equations (13) $)_{1},(13)_{2}$ and (14) $)_{1}-(14)_{4}$, as well as (15) 1 , and thanks to the divergence theorem we reach the following relation, valid for $t \geq 0$ :

$$
\begin{aligned}
& 2 \int_{B}\left[\rho u_{i}^{*}(t) \dot{u}_{i}^{*}(t)+\rho \chi \varphi^{*}(t) \dot{\varphi}^{*}(t)\right] d v \\
& =\int_{0}^{t} \int_{B}\left[\dot{\alpha}^{*}(t-s) \rho \eta^{*}(t+s)-\dot{\alpha}^{*}(t+s) \rho \eta^{*}(t-s)\right] d v d s .
\end{aligned}
$$

Integrating then Equation (13) 3 , considering Equation $(15)_{2}$ and applying again the divergence theorem we get

$$
\begin{aligned}
& 2 \int_{B}\left[\rho u_{i}^{*}(t) \dot{u}_{i}^{*}(t)+\rho \chi \varphi^{*}(t) \dot{\varphi}^{*}(t)\right] d v \\
& =\frac{1}{T_{0}} \int_{0}^{t} \int_{B}\left[\dot{\beta}_{i}^{*}(t+s) \int_{0}^{t-s} q_{i}^{*}(z) d z-\dot{\beta}_{i}^{*}(t-s) \int_{0}^{t+s} q_{i}^{*}(z) d z\right] d v d s .
\end{aligned}
$$

The equation that remains to be employed at this point is the $(14)_{5}$ which, together with def. (11), leads to

$$
\begin{aligned}
& \frac{d}{d t} \int_{B}\left[\rho u_{i}^{*}(t) u_{i}^{*}(t)+\rho \chi\left(\varphi^{*}\right)^{2}(t)\right] d v+\frac{2}{T_{0}} \int_{B} K_{i j} \overline{\bar{\beta}}_{j}(t) \overline{\bar{\beta}}_{i}(t) d v+\frac{2 \tau_{q}}{T_{0}} \int_{B} K_{i j} \overline{\bar{\beta}}_{j}(t) \bar{\beta}_{i}(t) d v \\
& +\frac{\tau_{q}^{2}}{T_{0}} \int_{B} K_{i j} \overline{\bar{\beta}}_{j}(t) \beta_{i}(t) d v+\frac{1}{T_{0}} \int_{B}\left[k_{i j}+\left(\tau_{\alpha}+\tau_{q}\right) K_{i j}\right] \overline{\bar{\beta}}_{j}(t) \overline{\bar{\beta}}_{i}(t) d v \\
& +\frac{\tau_{q}}{T_{0}} \int_{B}\left[2 k_{i j}+\left(2 \tau_{\alpha}+\tau_{q}\right) K_{i j}\right] \overline{\bar{\beta}}_{j}(t) \bar{\beta}_{i}(t) d v+\frac{\tau_{q}^{2}}{T_{0}} \int_{B}\left(k_{i j}+\tau_{\alpha} K_{i j}\right) \overline{\bar{\beta}}_{j}(t) \beta_{i}(t) d v \\
& +\frac{\tau_{q}}{2 T_{0}} \int_{B}\left[\left(\tau_{q}+2 \tau_{T}\right) k_{i j}+\tau_{\alpha} \tau_{q} K_{i j}\right] \bar{\beta}_{j}(t) \bar{\beta}_{i}(t) d v+\frac{\tau_{T} \tau_{q}^{2}}{T_{0}} \int_{B} k_{i j} \bar{\beta}_{j}(t) \beta_{i}(t) d v=0,
\end{aligned}
$$

where appropriate rearrangements have been performed similarly to what was done in [12]. Now, properly handling the time derivatives and the notation (10), and subsequently integrating three times over $t$, we are led to

$$
\begin{aligned}
& \int_{0}^{t} \int_{0}^{s} \int_{B}\left[\rho u_{i}^{*}(z) u_{i}^{*}(z)+\rho \chi\left(\varphi^{*}\right)^{2}(z)\right] d v d z d s+\frac{1}{T_{0}} \int_{0}^{t} \int_{0}^{s} \int_{B} K_{i j} \overline{\bar{\beta}}_{j}(z) \overline{\bar{\beta}}_{i}(z) d v d z d s \\
& +\frac{\tau_{q}}{T_{0}} \int_{0}^{t} \int_{B} K_{i j} \overline{\bar{\beta}}_{j}(s) \overline{\bar{\beta}}_{i}(s) d v d s+\frac{\tau_{q}^{2}}{2 T_{0}} \int_{B} K_{i j} \overline{\bar{\beta}}_{j}(t) \overline{\bar{\beta}}_{i}(t) d v \\
& +\frac{1}{T_{0}} \int_{0}^{t} \int_{0}^{s} \int_{0}^{z} \int_{B} \varkappa_{i j} \overline{\bar{\beta}}_{j}(r) \overline{\bar{\beta}}_{i}(r) d v d r d z d s+\frac{\tau_{q}}{T_{0}} \int_{0}^{t} \int_{0}^{s} \int_{B} \varkappa_{i j} \overline{\bar{\beta}}_{j}(z) \overline{\bar{\beta}}_{i}(z) d v d z d s \\
& +\frac{\tau_{q}^{2}}{2 T_{0}} \int_{0}^{t} \int_{B} \gamma_{i j} \overline{\bar{\beta}}_{j}(s) \overline{\bar{\beta}}_{i}(s) d v d s+\frac{\tau_{T} \tau_{q}^{2}}{2 T_{0}} \int_{0}^{t} \int_{0}^{s} \int_{B} k_{i j} \bar{\beta}_{j}(z) \bar{\beta}_{i}(z) d v d z d s \\
& +\frac{\tau_{q}}{T_{0}} \int_{0}^{t} \int_{0}^{s} \int_{0}^{z} \int_{B} \kappa_{i j} \bar{\beta}_{j}(r) \bar{\beta}_{i}(r) d v d r d z d s=0
\end{aligned}
$$


that actually coincides with Equation (6.9) of [34] (p. 384) except for the term related to the presence of porosity and where, for current and future needs of compactness, we have defined:

$$
\varkappa_{i j}=k_{i j}+\left(\tau_{\alpha}-\tau_{q}\right) K_{i j}, \quad \gamma_{i j}=k_{i j}+\tau_{\alpha} K_{i j}, \quad \kappa_{i j}=\tau_{T} k_{i j}-\frac{\tau_{q}}{2}\left(k_{i j}+\tau_{\alpha} K_{i j}\right),
$$

which clearly result symmetric under the conditions (6). So, once again, under the constitutive hypotheses of Theorem 1, all the integral terms in the LHS of Equation (24) are not negative, except for the last integral of indefinite sign. It follows that, for each $t \geq 0$,

$$
\frac{\tau_{T} \tau_{q}^{2}}{2 T_{0}} \int_{0}^{t} \int_{0}^{s} \int_{B} k_{i j} \bar{\beta}_{j}(z) \bar{\beta}_{i}(z) d v d z d s+\frac{\tau_{q}}{T_{0}} \int_{0}^{t} \int_{0}^{s} \int_{0}^{z} \int_{B} \kappa_{i j} \bar{\beta}_{j}(r) \bar{\beta}_{i}(r) d v d r d z d s \leq 0,
$$

and therefore a Gronwall inequality can be implemented exactly as in [34] (pp. 385-386): analogous considerations, that therefore we only cite here, lead to the first desired result of uniqueness.

Remark 1. As an alternative, starting again from (24), we could also directly achieve the result by strengthening the hypotheses of the Theorem 1. In particular, one could require in addition the positive semi-definiteness of the tensors $\varkappa_{i j}$ and $\kappa_{i j}$, in such a way to guarantee the thermodynamic compatibility (TC) of the time-differential three-phase lag model, see [35] (p. 228).

Feeling that, from an operational point of view, the proof of the uniqueness of the solution proposed in this section is very similar to what shown in [34], we will follow in the Section 5 a different path in order to prove once again such a result, this time using the logarithmic convexity method.

\section{Second Result: Continuous Dependence of the Solution With Respect to External Data}

With the intent to go ahead in the verification of the well-posedness for the linear thermoelastic model under investigation, we approach here the continuous dependence question. We start from the changed initial-boundary value problem $\mathscr{P}^{*}$ and assume only trivial boundary conditions, i.e., we select $\mathscr{D}=\left\{f_{i}, l, s ; u_{i}^{0}, \dot{u}_{i}^{0}, \varphi^{0}, \dot{\varphi}^{0}, T^{0}, q_{i}^{0}, \dot{q}_{i}^{0} ; 0,0,0,0,0,0\right\}$. We then proceed by illustrating the procedure followed. Integrating the products of Equation $(13)_{1}$ by $\dot{u}_{i}^{*}(t)$ and of Equation $(13)_{2}$ by $\dot{\varphi}^{*}(t)$ over the volume $B$, taking into account the null boundary data and the Equation $(15)_{1}$ and using the divergence theorem we get:

$$
\begin{aligned}
& \frac{1}{2} \frac{d}{d t} \int_{B}\left[\rho \frac{\partial u_{i}^{*}}{\partial t} \frac{\partial u_{i}^{*}}{\partial t}+\rho \chi\left(\frac{\partial \varphi^{*}}{\partial t}\right)^{2}\right] d v \\
& =\int_{B}\left(F_{i} \frac{\partial u_{i}^{*}}{\partial t}+L \frac{\partial \varphi^{*}}{\partial t}\right) d v-\int_{B}\left(t_{i j}^{*} \frac{\partial e_{i j}^{*}}{\partial t}+h_{i}^{*} \frac{\partial \varphi_{, i}^{*}}{\partial t}-g^{*} \frac{\partial \varphi^{*}}{\partial t}\right) d v
\end{aligned}
$$

Referring now to the constitutive Equations $(14)_{1}-(14)_{4}$ and starting from Equation (27) we can write in a way similar to what done in [26]:

$$
\begin{aligned}
& \frac{1}{2} \frac{d}{d t} \int_{B}\left[\rho \frac{\partial u_{i}^{*}}{\partial t} \frac{\partial u_{i}^{*}}{\partial t}+\rho \chi\left(\frac{\partial \varphi^{*}}{\partial t}\right)^{2}+C_{i j k l} e_{i j}^{*} e_{k l}^{*}+A_{i j} \varphi_{, i}^{*} \varphi_{, j}^{*}+\xi\left(\varphi^{*}\right)^{2}+a\left(\frac{\partial \alpha^{*}}{\partial t}\right)^{2}\right] d v \\
& =\int_{B}\left(F_{i} \frac{\partial u_{i}^{*}}{\partial t}+L \frac{\partial \varphi^{*}}{\partial t}\right) d v+\int_{B} \rho \frac{\partial \eta^{*}}{\partial t} \frac{\partial \alpha^{*}}{\partial t} d v \\
& -\int_{B}\left[B_{i j}\left(\varphi^{*} \frac{\partial e_{i j}^{*}}{\partial t}+e_{i j}^{*} \frac{\partial \varphi^{*}}{\partial t}\right)+D_{i j k}\left(\varphi_{, k}^{*} \frac{\partial e_{i j}^{*}}{\partial t}+e_{i j}^{*} \frac{\partial \varphi_{, k}^{*}}{\partial t}\right)+d_{i}\left(\varphi^{*} \frac{\partial \varphi_{, i}^{*}}{\partial t}+\varphi_{, i}^{*} \frac{\partial \varphi^{*}}{\partial t}\right)\right] d v
\end{aligned}
$$


Then we introduce the Equations $(13)_{3},(14)_{5}$ and $(15)_{2}$, apply the divergence theorem again with null boundary conditions and remember the definition of the (positive definite) energy $W^{*}$ associated with the strain and volume distortion, as deduced from Ieşan [29], in order to write:

$$
\begin{aligned}
& \frac{1}{2} \frac{d}{d t} \int_{B}\left[\rho \frac{\partial u_{i}^{*}}{\partial t} \frac{\partial u_{i}^{*}}{\partial t}+\rho \chi\left(\frac{\partial \varphi^{*}}{\partial t}\right)^{2}+2 W^{*}+a\left(\frac{\partial \alpha^{*}}{\partial t}\right)^{2}\right] d v \\
& =\int_{B}\left[F_{i} \frac{\partial u_{i}^{*}}{\partial t}+L \frac{\partial \varphi^{*}}{\partial t}+\frac{S}{T_{0}} \frac{\partial \alpha^{*}}{\partial t}-\frac{\Omega_{i}}{T_{0}} \frac{\partial \beta_{i}^{*}}{\partial t}\right] d v \\
& -\frac{1}{T_{0}} \int_{B}\left(K_{i j} \overline{\bar{\beta}}_{j}+\gamma_{i j} \bar{\beta}_{j}+\tau_{T} k_{i j} \beta_{j}\right)\left(\bar{\beta}_{i}+\tau_{q} \beta_{i}+\frac{\tau_{q}^{2}}{2} \frac{\partial \beta_{i}}{\partial t}\right) d v
\end{aligned}
$$

where the following notation has been used

$$
W^{*}(\mathbf{x}, t)=\frac{1}{2} C_{i j k l} e_{i j}^{*} e_{k l}^{*}+\frac{1}{2} \xi\left(\varphi^{*}\right)^{2}+\frac{1}{2} A_{i j} \varphi_{, i}^{*} \varphi_{, j}^{*}+B_{i j} e_{i j}^{*} \varphi^{*}+D_{i j k} e_{i j}^{*} \varphi_{, k}^{*}+d_{i} \varphi_{, i}^{*} \varphi^{*},
$$

and where we can note that

$$
\frac{\partial \beta_{i}^{*}}{\partial t}=\left(\bar{\beta}_{i}+\tau_{q} \beta_{i}+\frac{\tau_{q}^{2}}{2} \frac{\partial \beta_{i}}{\partial t}\right) .
$$

Working with the integral operator (10) and remembering the def. (25), after straightforward calculations we are led from the Equation (28) to

$$
\begin{aligned}
& \frac{1}{2} \frac{d}{d t} \int_{B}\left[\rho \frac{\partial u_{i}^{*}}{\partial t} \frac{\partial u_{i}^{*}}{\partial t}+\rho \chi\left(\frac{\partial \varphi^{*}}{\partial t}\right)^{2}+2 W^{*}+a\left(\frac{\partial \alpha^{*}}{\partial t}\right)^{2}\right] d v+\frac{\tau_{q}^{2}}{4 T_{0}} \frac{d^{3}}{d t^{3}} \int_{B} K_{i j} \overline{\bar{\beta}}_{j} \overline{\bar{\beta}}_{i} d v \\
& +\frac{\tau_{q}}{2 T_{0}} \frac{d^{2}}{d t^{2}} \int_{B} K_{i j} \overline{\bar{\beta}}_{j} \overline{\bar{\beta}}_{i} d v+\frac{1}{2 T_{0}} \frac{d}{d t} \int_{B} K_{i j} \overline{\bar{\beta}}_{j} \overline{\bar{\beta}}_{i} d v+\frac{\tau_{q}^{2}}{4 T_{0}} \frac{d^{2}}{d t^{2}} \int_{B} \gamma_{i j} \bar{\beta}_{j} \bar{\beta}_{i} d v \\
& +\frac{1}{4 T_{0}} \frac{d}{d t} \int_{B}\left(2 \kappa_{i j}+3 \tau_{q} \varkappa_{i j}\right) \bar{\beta}_{j} \bar{\beta}_{i} d v+\frac{1}{T_{0}} \int_{B} \varkappa_{i j} \bar{\beta}_{j} \bar{\beta}_{i} d v+\frac{\tau_{T} \tau_{q}^{2}}{4 T_{0}} \frac{d}{d t} \int_{B} \kappa_{i j} \beta_{j} \beta_{i} d v+\frac{\tau_{q}}{T_{0}} \int_{B} \kappa_{i j} \beta_{j} \beta_{i} d v \\
& =\int_{B}\left[F_{i} \frac{\partial u_{i}^{*}}{\partial t}+L \frac{\partial \varphi^{*}}{\partial t}+\frac{S}{T_{0}} \frac{\partial \alpha^{*}}{\partial t}-\frac{\Omega_{i}}{T_{0}} \frac{\partial \beta_{i}^{*}}{\partial t}\right] d v .
\end{aligned}
$$

Emphasizing that the initial data selected are not null in this case, we proceed with three integrations in the time variable, obtaining:

$$
\begin{aligned}
& \frac{1}{2} \int_{0}^{t} \int_{0}^{s} \int_{B}\left[\rho \frac{\partial u_{i}^{*}}{\partial z} \frac{\partial u_{i}^{*}}{\partial z}+\rho \chi\left(\frac{\partial \varphi^{*}}{\partial z}\right)^{2}+2 W^{*}+a\left(\frac{\partial \alpha^{*}}{\partial z}\right)^{2}\right] d v d z d s+\frac{\tau_{q}^{2}}{4 T_{0}} \int_{B} K_{i j} \overline{\bar{\beta}}_{j} \overline{\bar{\beta}}_{i} d v \\
& +\frac{\tau_{q}}{2 T_{0}} \int_{0}^{t} \int_{B} K_{i j} \overline{\bar{\beta}}_{j} \overline{\bar{\beta}}_{i} d v d s+\frac{1}{2 T_{0}} \int_{0}^{t} \int_{0}^{s} \int_{B} K_{i j} \overline{\bar{\beta}}_{j} \overline{\bar{\beta}}_{i} d v d z d s+\frac{\tau_{q}^{2}}{4 T_{0}} \int_{0}^{t} \int_{B} \gamma_{i j} \bar{\beta}_{j} \bar{\beta}_{i} d v d s \\
& +\frac{1}{4 T_{0}} \int_{0}^{t} \int_{0}^{s} \int_{B}\left(2 \kappa_{i j}+3 \tau_{q} \varkappa_{i j}\right) \bar{\beta}_{j} \bar{\beta}_{i} d v d z d s+\frac{1}{T_{0}} \int_{0}^{t} \int_{0}^{s} \int_{0}^{z} \int_{B} \varkappa_{i j} \bar{\beta}_{j} \bar{\beta}_{i} d v d r d z d s \\
& +\frac{\tau_{T} \tau_{q}^{2}}{4 T_{0}} \int_{0}^{t} \int_{0}^{s} \int_{B} k_{i j} \beta_{j} \beta_{i} d v d z d s+\frac{\tau_{q}}{T_{0}} \int_{0}^{t} \int_{0}^{s} \int_{0}^{z} \int_{B} \kappa_{i j} \beta_{j} \beta_{i} d v d r d z d s \\
& =\frac{t^{2}}{4} \int_{B}\left[\rho \frac{\partial u_{i}^{*}}{\partial t}(0) \frac{\partial u_{i}^{*}}{\partial t}(0)+\rho \chi\left(\frac{\partial \varphi^{*}}{\partial t}(0)\right)^{2}+2 W^{*}(0)+a\left(\frac{\partial \alpha^{*}}{\partial t}(0)\right)^{2}\right] d v \\
& +\int_{0}^{t} \int_{0}^{s} \int_{0}^{z} \int_{B}\left[F_{i} \frac{\partial u_{i}^{*}}{\partial r}+L \frac{\partial \varphi^{*}}{\partial r}+\frac{s}{T_{0}} \frac{\partial \alpha^{*}}{\partial r}-\frac{\Omega_{i}}{T_{0}} \frac{\partial \beta_{i}^{*}}{\partial r}\right] d v d r d z d s,
\end{aligned}
$$

a relation from which we will derive a conservation law in Section 5 , in the case of cancellation of all external data for a further research of the uniqueness. 
In analogy with [26] and only for convenience related to possible future comparisons, we define $\Gamma_{i}(\mathbf{x}, t)=-\Omega_{i}(\mathbf{x}, t)$, and again we underline that the term $\partial \beta_{i} / \partial t$ that flows from the expression (30) of $\partial \beta_{i}^{*} / \partial t$ does not have a suitable counterpart in the LHS of Equation (32). An integration by parts is then necessary:

$$
\int_{0}^{t} \int_{0}^{s} \int_{0}^{z} \int_{B} \Gamma_{i} \frac{\partial \beta_{i}}{\partial r} d v d r d z d s=\int_{0}^{t} \int_{0}^{s} \int_{B} \Gamma_{i} \beta_{i} d v d z d s-\int_{0}^{t} \int_{0}^{s} \int_{0}^{z} \int_{B} \frac{\partial \Gamma_{i}}{\partial r} \beta_{i} d v d r d z d s
$$

and, taking as a reference the term of Equation (32)

$$
\frac{\tau_{T} \tau_{q}^{2}}{4 T_{0}} \int_{0}^{t} \int_{0}^{s} \int_{B} k_{i j} \beta_{j} \beta_{i} d v d z d s
$$

we evaluate using the arithmetic-geometric mean inequality the first addend at RHS of (33).

In general it turns out to be $A B \leq A^{2} /(2 \varepsilon)+\varepsilon B^{2} / 2 \forall \varepsilon \in \mathbb{R}^{+}$, and we select for our purposes $\varepsilon=\tau_{T} k_{m} / 2$ (where $k_{m}$ is a scalar related to the lowest eigenvalue of $k_{i j}$ ). We underline that $k_{i j}$ is assumed to be a positive definite tensor. Then from the Equation (33) multiplied by $\tau_{q}^{2} /\left(2 T_{0}\right)$ the following estimate can be deduced:

$$
\begin{aligned}
& \frac{\tau_{q}^{2}}{2 T_{0}} \int_{0}^{t} \int_{0}^{s} \int_{0}^{z} \int_{B} \Gamma_{i} \frac{\partial \beta_{i}}{\partial r} d v d r d z d s \leq \frac{\tau_{q}^{2}}{2 \tau_{T} T_{0}} \int_{0}^{t} \int_{0}^{s} \int_{B} \frac{\Gamma_{i} \Gamma_{i}}{k_{m}} d v d z d s+\frac{\tau_{T} \tau_{q}^{2}}{8 T_{0}} \int_{0}^{t} \int_{0}^{s} \int_{B} k_{i j} \beta_{j} \beta_{i} d v d z d s \\
& -\frac{\tau_{q}^{2}}{2 T_{0}} \int_{0}^{t} \int_{0}^{s} \int_{0}^{z} \int_{B} \frac{\partial \Gamma_{i}}{\partial r} \beta_{i} d v d r d z d s .
\end{aligned}
$$

At this point, starting from the Equation (32) it is convenient to propose the relation that follows:

$$
\begin{aligned}
& \frac{1}{2} \int_{0}^{t} \int_{0}^{s} \int_{B}\left[\rho \frac{\partial u_{i}^{*}}{\partial z} \frac{\partial u_{i}^{*}}{\partial z}+\rho \chi\left(\frac{\partial \varphi^{*}}{\partial z}\right)^{2}+2 W^{*}+a\left(\frac{\partial \alpha^{*}}{\partial z}\right)^{2}\right] d v d z d s+\frac{\tau_{q}^{2}}{4 T_{0}} \int_{B} K_{i j} \overline{\bar{\beta}}_{j} \overline{\bar{\beta}}_{i} d v \\
& +\frac{\tau_{q}}{2 T_{0}} \int_{0}^{t} \int_{B} K_{i j} \overline{\bar{\beta}}_{j} \overline{\bar{\beta}}_{i} d v d s+\frac{1}{2 T_{0}} \int_{0}^{t} \int_{0}^{s} \int_{B} K_{i j} \overline{\bar{\beta}}_{j} \overline{\bar{\beta}}_{i} d v d z d s+\frac{\tau_{q}^{2}}{4 T_{0}} \int_{0}^{t} \int_{B} \gamma_{i j} \bar{\beta}_{j} \bar{\beta}_{i} d v d s \\
& +\frac{1}{2 T_{0}} \int_{0}^{t} \int_{0}^{s} \int_{B}\left(\tau_{T} k_{i j}+\tau_{q} \gamma_{i j}\right) \bar{\beta}_{j} \bar{\beta}_{i} d v d z d s+\frac{1}{T_{0}} \int_{0}^{t} \int_{0}^{s} \int_{0}^{z} \int_{B} \gamma_{i j} \bar{\beta}_{j} \bar{\beta}_{i} d v d r d z d s \\
& +\frac{\tau_{T} \tau_{q}^{2}}{8 T_{0}} \int_{0}^{t} \int_{0}^{s} \int_{B} k_{i j} \beta_{j} \beta_{i} d v d z d s+\frac{\tau_{T} \tau_{q}}{T_{0}} \int_{0}^{t} \int_{0}^{s} \int_{0}^{z} \int_{B} k_{i j} \beta_{j} \beta_{i} d v d r d z d s \\
& \leq \frac{t^{2}}{4} \int_{B}\left[\rho \frac{\partial u_{i}^{*}}{\partial t}(0) \frac{\partial u_{i}^{*}}{\partial t}(0)+\rho \chi\left(\frac{\partial \varphi^{*}}{\partial t}(0)\right)^{2}+2 W^{*}(0)+a\left(\frac{\partial \alpha^{*}}{\partial t}(0)\right)^{2}\right] d v \\
& +\int_{0}^{t} \int_{0}^{s} \int_{0}^{z} \int_{B}\left[F_{i} \frac{\partial u_{i}^{*}}{\partial r}+L \frac{\partial \varphi^{*}}{\partial r}+\frac{s}{T_{0}} \frac{\partial \alpha^{*}}{\partial r}+\frac{\Gamma_{i}}{T_{0}} \bar{\beta}_{i}+\frac{\tau_{q}}{T_{0}}\left(\Gamma_{i}-\frac{\tau_{q}}{2} \frac{\partial \Gamma_{i}}{\partial r}\right) \beta_{i}\right] d v d r d z d s \\
& +\frac{\tau_{q}^{2}}{2 \tau_{T} T_{0}} \int_{0}^{t} \int_{0}^{s} \int_{B} \frac{\Gamma_{i} \Gamma_{i}}{k_{m}} d v d z d s+\frac{3 \tau_{q}^{2}}{4 T_{0}} \int_{0}^{t} \int_{0}^{s} \int_{B} K_{i j} \bar{\beta}_{j} \bar{\beta}_{i} d v d z d s \\
& +\frac{\tau_{q}}{T_{0}} \int_{0}^{t} \int_{0}^{s} \int_{0}^{z} \int_{B} K_{i j} \bar{\beta}_{j} \bar{\beta}_{i} d v d r d z d s+\frac{\tau_{q}^{2}}{2 T_{0}} \int_{0}^{t} \int_{0}^{s} \int_{0}^{z} \int_{B} \gamma_{i j} \beta_{j} \beta_{i} d v d r d z d s .
\end{aligned}
$$


Similarly to what has been said for $k_{m}$, let us call $K_{m}$ a scalar related to the smallest eigenvalue of $K_{i j}$. In this case, $K_{i j}$ is assumed to be a positive semi-definite tensor. We then make a suitable estimate of the following term, detectable in Equation (36), through the Cauchy-Schwarz inequality:

$$
\begin{aligned}
& \int_{0}^{t} \int_{0}^{s} \int_{0}^{z} \int_{B}\left[F_{i} \frac{\partial u_{i}^{*}}{\partial r}+L \frac{\partial \varphi^{*}}{\partial r}+\frac{S}{T_{0}} \frac{\partial \alpha^{*}}{\partial r}+\frac{\Gamma_{i}}{T_{0}} \bar{\beta}_{i}+\frac{\tau_{q}}{T_{0}}\left(\Gamma_{i}-\frac{\tau_{q}}{2} \frac{\partial \Gamma_{i}}{\partial r}\right) \beta_{i}\right] d v d r d z d s \leq \int_{0}^{t} g(s) \\
& \times\left\{\int_{0}^{s} \int_{0}^{z} \int_{B}\left[\rho \frac{\partial u_{i}^{*}}{\partial r} \frac{\partial u_{i}^{*}}{\partial r}+\rho \chi\left(\frac{\partial \varphi^{*}}{\partial r}\right)^{2}+a\left(\frac{\partial \alpha^{*}}{\partial r}\right)^{2}+\frac{\tau_{T} k_{i j}+\tau_{q} \gamma_{i j}}{T_{0}} \bar{\beta}_{j} \bar{\beta}_{i}+\frac{\tau_{T} \tau_{q}^{2}}{4 T_{0}} k_{i j} \beta_{j} \beta_{i}\right] d v d r d z\right\}^{1 / 2} d s,
\end{aligned}
$$

where

$$
\begin{aligned}
& g(t)=\left\{\int _ { 0 } ^ { t } \int _ { 0 } ^ { s } \int _ { B } \left[\frac{F_{i} F_{i}}{\rho}+\frac{L^{2}}{\rho \chi}+\frac{S^{2}}{a T_{0}^{2}}+\frac{\Gamma_{i} \Gamma_{i}}{T_{0}\left[\left(\tau_{T}+\tau_{q}\right) k_{m}+\tau_{q} \tau_{\alpha} K_{m}\right]}\right.\right. \\
& \left.\left.+\frac{4}{\tau_{T} T_{0} k_{m}}\left(\Gamma_{i}-\frac{\tau_{q}}{2} \frac{\partial \Gamma_{i}}{\partial z}\right)\left(\Gamma_{i}-\frac{\tau_{q}}{2} \frac{\partial \Gamma_{i}}{\partial z}\right)\right] d v d z d s\right\}^{1 / 2} .
\end{aligned}
$$

Remark 2. The Equations (37) and (38) actually lead back to their counterparts in [26] (see p. 1592) for a thermoelastic model with voids and only two relaxation times, namely $\tau_{q}$ and $\tau_{T}$ : such equations can be seen in fact as special cases of (37) and (38) when $\tau_{\alpha}=0$ is selected.

Now, from the LHS of Equation (36) we define the following functional $\mathscr{H}(t)$, which is not negative even without invoking the hypotheses of thermodynamic compatibility TC for the time-differential three-phase lag model:

$$
\begin{aligned}
& \mathscr{H}(t)=\frac{1}{2} \int_{0}^{t} \int_{0}^{s} \int_{B}\left[\rho \frac{\partial u_{i}^{*}}{\partial z} \frac{\partial u_{i}^{*}}{\partial z}+\rho \chi\left(\frac{\partial \varphi^{*}}{\partial z}\right)^{2}+2 W^{*}+a\left(\frac{\partial \alpha^{*}}{\partial z}\right)^{2}\right] d v d z d s+\frac{\tau_{q}^{2}}{4 T_{0}} \int_{B} K_{i j} \overline{\bar{\beta}}_{j} \overline{\bar{\beta}}_{i} d v \\
& +\frac{\tau_{q}}{2 T_{0}} \int_{0}^{t} \int_{B} K_{i j} \overline{\bar{\beta}}_{j} \overline{\bar{\beta}}_{i} d v d s+\frac{1}{2 T_{0}} \int_{0}^{t} \int_{0}^{s} \int_{B} K_{i j} \overline{\bar{\beta}}_{j} \overline{\bar{\beta}}_{i} d v d z d s+\frac{\tau_{q}^{2}}{4 T_{0}} \int_{0}^{t} \int_{B} \gamma_{i j} \bar{\beta}_{j} \bar{\beta}_{i} d v d s \\
& +\frac{1}{2 T_{0}} \int_{0}^{t} \int_{0}^{s} \int_{B}\left(\tau_{T} k_{i j}+\tau_{q} \gamma_{i j}\right) \bar{\beta}_{j} \bar{\beta}_{i} d v d z d s+\frac{1}{T_{0}} \int_{0}^{t} \int_{0}^{s} \int_{0}^{z} \int_{B} \gamma_{i j} \bar{\beta}_{j} \bar{\beta}_{i} d v d r d z d s \\
& +\frac{\tau_{T} \tau_{q}^{2}}{8 T_{0}} \int_{0}^{t} \int_{0}^{s} \int_{B} k_{i j} \beta_{j} \beta_{i} d v d z d s+\frac{\tau_{T} \tau_{q}}{T_{0}} \int_{0}^{t} \int_{0}^{s} \int_{0}^{z} \int_{B} k_{i j} \beta_{j} \beta_{i} d v d r d z d s .
\end{aligned}
$$

Remark 3. As an alternative, one could select a functional similar to Equation (7.1) of [34] (p. 387): however, this choice would involve the activation of the thermodynamic compatibility hypotheses TC.

From Equations (36), (37) and (39) we can proceed with the following estimate:

$$
\begin{aligned}
& \mathscr{H}(t) \leq \frac{t^{2}}{4} \int_{B}\left[\rho \frac{\partial u_{i}^{*}}{\partial t}(0) \frac{\partial u_{i}^{*}}{\partial t}(0)+\rho \chi\left(\frac{\partial \varphi^{*}}{\partial t}(0)\right)^{2}+2 W^{*}(0)+a\left(\frac{\partial \alpha^{*}}{\partial t}(0)\right)^{2}\right] d v \\
& +\frac{\tau_{q}^{2}}{2 \tau_{T} T_{0}} \int_{0}^{t} \int_{0}^{s} \int_{B} \frac{\Gamma_{i} \Gamma_{i}}{k_{m}} d v d z d s+\frac{3 \tau_{q}^{2}}{4 T_{0}} \int_{0}^{t} \int_{0}^{s} \int_{B} K_{i j} \bar{\beta}_{j} \bar{\beta}_{i} d v d z d s \\
& +\frac{\tau_{q}}{T_{0}} \int_{0}^{t} \int_{0}^{s} \int_{0}^{z} \int_{B} K_{i j} \bar{\beta}_{j} \bar{\beta}_{i} d v d r d z d s+\frac{\tau_{q}^{2}}{2 T_{0}} \int_{0}^{t} \int_{0}^{s} \int_{0}^{z} \int_{B} \gamma_{i j} \beta_{j} \beta_{i} d v d r d z d s+\int_{0}^{t} g(s) \sqrt{2 \mathscr{H}(s)} d s,
\end{aligned}
$$


which can be further rearranged in:

$$
\begin{aligned}
& \mathscr{H}(t) \leq \frac{t^{2}}{4} \int_{B}\left[\rho \frac{\partial u_{i}^{*}}{\partial t}(0) \frac{\partial u_{i}^{*}}{\partial t}(0)+\rho \chi\left(\frac{\partial \varphi^{*}}{\partial t}(0)\right)^{2}+2 W^{*}(0)+a\left(\frac{\partial \alpha^{*}}{\partial t}(0)\right)^{2}\right] d v \\
& +\frac{\tau_{q}^{2}}{2 \tau_{T} T_{0}} \int_{0}^{t} \int_{0}^{s} \int_{B} \frac{\Gamma_{i} \Gamma_{i}}{k_{m}} d v d z d s+\int_{0}^{t} g(s) \sqrt{2 \mathscr{H}(s)} d s+\frac{2}{\delta} \int_{0}^{t} \mathscr{H}(s) d s
\end{aligned}
$$

having defined

$$
\frac{2}{\delta}=\max \left\{\frac{3}{\tau_{\alpha}}, \frac{4}{\tau_{T}} \max \frac{\sqrt{\gamma_{r s} \gamma_{r s}}}{k_{m}}\right\}
$$

The theorem about continuous dependence follows along with its proof.

Theorem 2. Let us consider the problem $\mathscr{P}$ characterized by trivial boundary data, or in other words let $\mathscr{D}=\left\{f_{i}, l, s ; u_{i}^{0}, \dot{u}_{i}^{0}, \varphi^{0}, \dot{\varphi}^{0}, T^{0}, q_{i}^{0}, \dot{q}_{i}^{0} ; 0,0,0,0,0,0\right\}$. Let also $\mathscr{S}=\left\{u_{i}, \varphi, \alpha, e_{i j}, \beta_{j}, t_{i j}, h_{i}, g, \eta, q_{i}\right\}(\mathbf{x}, t)$ be its solution. Moreover, let us assume the validity of the following hypotheses:

- $\rho, \chi$ and a strictly positive;

- $W^{*}$ positive definite quadratic form in the variables $e_{i j}^{*}, \varphi^{*}, \varphi_{, i}^{*}$;

- $k_{i j}$ positive definite tensor and $K_{i j}$ positive semi-definite tensor.

Then, for any determined and finite time $t_{f} \in(0, \infty)$, the validity of the estimate that follows is guaranteed:

$$
\sqrt{\mathscr{H}(t)} \leq Z(0) e^{t / \delta}+\frac{1}{\sqrt{2}} \int_{0}^{t} g(s) e^{(t-s) / \delta} d s, \quad t \in\left[0, t_{f}\right]
$$

where $\mathscr{H}(t)$ is given by the Equation (39), $g(t)$ is defined through the Equation (38) and

$$
\begin{aligned}
& Z(t)=\left\{\frac{2}{\delta} \int_{0}^{t} \mathscr{H}(s) d s+\int_{0}^{t} g(s) \sqrt{2 \mathscr{H}(s)} d s+\frac{\tau_{q}^{2}}{2 \tau_{T} T_{0}} \int_{0}^{t_{f}} \int_{0}^{s} \int_{B} \frac{\Gamma_{i} \Gamma_{i}}{k_{m}} d v d z d s\right. \\
& \left.+\frac{t_{f}^{2}}{4} \int_{B}\left[\rho \frac{\partial u_{i}^{*}}{\partial t}(0) \frac{\partial u_{i}^{*}}{\partial t}(0)+\rho \chi\left(\frac{\partial \varphi^{*}}{\partial t}(0)\right)^{2}+2 W^{*}(0)+a\left(\frac{\partial \alpha^{*}}{\partial t}(0)\right)^{2}\right] d v\right\}^{1 / 2} .
\end{aligned}
$$

Proof of Theorem 2. If we bound $t \in\left[0, t_{f}\right]$, then from Equations (41) and (44) we deduce that $\mathscr{H}(t) \leq Z^{2}(t)$ and also $\sqrt{\mathscr{H}(t)} \leq Z(t)$. However, from (44) it follows also that:

$$
\frac{d Z^{2}}{d t}(t)=\frac{2}{\delta} \mathscr{H}(t)+\sqrt{2} g(t) \sqrt{\mathscr{H}(t)} \leq \frac{2}{\delta} Z^{2}(t)+\sqrt{2} g(t) Z(t)
$$

and so

$$
\frac{d Z}{d t}(t)-\frac{1}{\delta} Z(t) \leq \frac{g(t)}{\sqrt{2}} .
$$

If we multiply both members of the previous relation by $e^{-t / \delta}$ we get

$$
\frac{d}{d t}\left[Z(t) e^{-t / \delta}\right] \leq \frac{g(t)}{\sqrt{2}} e^{-t / \delta},
$$

on which we proceed with an integration from 0 to $t$, where $t$ is still bounded in $\left[0, t_{f}\right]$. Multiplying what we get by $e^{t / \delta}$ we readily come to the desired continuous dependence estimate (43). 


\section{Third Result: Verification of the Uniqueness Through the Logarithmic Convexity Method}

Let us define, starting from the Equation (24), the functional $\mathscr{F}\left(\mathscr{S}^{*}\right)$ associated with the solution $\mathscr{S}^{*}$ of the initial-boundary value problem at issue, in order to show that it is a logarithmically convex function of time; this will imply the uniqueness of the solution (see e.g., [36] and the references therein; see also Knops and Payne [37] and Ames and Straughan [38]):

$$
\begin{aligned}
& \mathscr{F}\left(\mathscr{S}^{*}\right)(t)=\int_{0}^{t} \int_{0}^{s} \int_{B}\left[\rho u_{i}^{*} u_{i}^{*}+\rho \chi\left(\varphi^{*}\right)^{2}\right] d v d z d s+\frac{1}{T_{0}} \int_{0}^{t} \int_{0}^{s} \int_{B} K_{i j} \overline{\bar{\beta}}_{j} \overline{\bar{\beta}}_{i} d v d z d s \\
& +\frac{\tau_{q}}{T_{0}} \int_{0}^{t} \int_{B} K_{i j} \overline{\bar{\beta}}_{j} \overline{\bar{\beta}}_{i} d v d s+\frac{\tau_{q}^{2}}{2 T_{0}} \int_{B} K_{i j} \overline{\overline{\bar{\beta}}}_{j} \overline{\bar{\beta}}_{i} d v+\frac{1}{T_{0}} \int_{0}^{t} \int_{0}^{s} \int_{0}^{z} \int_{B} \varkappa_{i j} \overline{\bar{\beta}}_{j} \overline{\bar{\beta}}_{i} d v d r d z d s \\
& +\frac{\tau_{q}}{T_{0}} \int_{0}^{t} \int_{0}^{s} \int_{B} \varkappa_{i j} \overline{\bar{\beta}}_{j} \overline{\bar{\beta}}_{i} d v d z d s+\frac{\tau_{q}^{2}}{2 T_{0}} \int_{0}^{t} \int_{B} \gamma_{i j} \overline{\bar{\beta}}_{j} \overline{\bar{\beta}}_{i} d v d s \\
& +\frac{\tau_{T} \tau_{q}^{2}}{2 T_{0}} \int_{0}^{t} \int_{0}^{s} \int_{B} k_{i j} \bar{\beta}_{j} \bar{\beta}_{i} d v d z d s+\frac{\tau_{q}}{T_{0}} \int_{0}^{t} \int_{0}^{s} \int_{0}^{z} \int_{B} \kappa_{i j} \bar{\beta}_{j} \bar{\beta}_{i} d v d r d z d s, \quad \forall t \geq 0 .
\end{aligned}
$$

We recall that if a not negative function is logarithmically convex, then it is either identically null or it is strictly positive, and in this case its logarithm is a convex function.

Remark 4. Under the thermodynamic compatibility (TC) conditions about the tensors $\varkappa_{i j}$ and $\kappa_{i j}$, assuming again $k_{i j}$ positive definite and $K_{i j}$ positive semi-definite, if $\rho>0$ and $\chi>0$, if the symmetry relations (6) are valid and if all the relaxation times are positive, then $\mathscr{F}\left(\mathscr{S}^{*}\right)(t) \geq 0 \forall t \geq 0$; moreover, the condition $\mathscr{F}\left(\mathscr{S}^{*}\right)(t)=0 \forall t \geq 0$ implies the null solution. Therefore, one can take $\mathscr{F}\left(\mathscr{S}^{*}\right)(t)$ as a possible measure of the solution $\mathscr{S}^{*}$ to the problem $\mathscr{P}^{*}$.

We take into account the modified initial-boundary value problem $\mathscr{P}^{*}$, once again with null assigned data $\mathscr{D}$. In this case, the dot notation for the time derivative can be employed without ambiguity since null initial data are considered. Working on the def. (45) through integrations by parts, invoking null initial conditions and remembering the symmetry of the tensors involved, we come to the following time derivative of the functional $\mathscr{F}\left(\mathscr{S}^{*}\right)(t)$ :

$$
\begin{aligned}
& \frac{d \mathscr{F}\left(\mathscr{S}^{*}\right)}{d t}(t)=2 \int_{0}^{t} \int_{0}^{s} \int_{B}\left(\rho u_{i}^{*} \dot{u}_{i}^{*}+\rho \chi \varphi^{*} \dot{\varphi}^{*}\right) d v d z d s+\frac{2}{T_{0}} \int_{0}^{t} \int_{0}^{s} \int_{B} K_{i j} \overline{\bar{\beta}}_{j} \overline{\bar{\beta}}_{i} d v d z d s \\
& +\frac{2 \tau_{q}}{T_{0}} \int_{0}^{t} \int_{B} K_{i j} \overline{\bar{\beta}}_{j} \overline{\bar{\beta}}_{i} d v d s+\frac{\tau_{q}^{2}}{T_{0}} \int_{B} K_{i j} \overline{\bar{\beta}}_{j} \overline{\bar{\beta}}_{i} d v+\frac{2}{T_{0}} \int_{0}^{t} \int_{0}^{s} \int_{0}^{z} \int_{B} \varkappa_{i j} \overline{\bar{\beta}}_{j} \bar{\beta}_{i} d v d r d z d s \\
& +\frac{2 \tau_{q}}{T_{0}} \int_{0}^{t} \int_{0}^{s} \int_{B} \varkappa_{i j} \overline{\bar{\beta}}_{j} \bar{\beta}_{i} d v d z d s+\frac{\tau_{q}^{2}}{T_{0}} \int_{0}^{t} \int_{B} \gamma_{i j} \overline{\bar{\beta}}_{j} \bar{\beta}_{i} d v d s \\
& +\frac{\tau_{T} \tau_{q}^{2}}{T_{0}} \int_{0}^{t} \int_{0}^{s} \int_{B} k_{i j} \bar{\beta}_{j} \beta_{i} d v d z d s+\frac{2 \tau_{q}}{T_{0}} \int_{0}^{t} \int_{0}^{s} \int_{0}^{z} \int_{B} \kappa_{i j} \bar{\beta}_{j} \beta_{i} d v d r d z d s, \quad \forall t \geq 0 .
\end{aligned}
$$

Preliminarily handling the Equation (46) through further integrations by parts, a new differentiation gives:

$$
\begin{aligned}
& \frac{d^{2} \mathscr{F}\left(\mathscr{S}^{*}\right)}{d t^{2}}(t)=2 \int_{0}^{t} \int_{0}^{s} \int_{B}\left[\rho u_{i}^{*} \dot{u}_{i}^{*}+\rho \chi\left(\dot{\varphi}^{*}\right)^{2}\right] d v d z d s+2 \int_{0}^{t} \int_{0}^{s} \int_{B}\left(\rho u_{i}^{*} \ddot{u}_{i}^{*}+\rho \chi \varphi^{*} \ddot{\varphi}^{*}\right) d v d z d s \\
& +\frac{2}{T_{0}} \int_{0}^{t} \int_{B} K_{i j} \overline{\bar{\beta}}_{j} \overline{\bar{\beta}}_{i} d v d s+\frac{2 \tau_{q}}{T_{0}} \int_{B} K_{i j} \overline{\bar{\beta}}_{j} \overline{\bar{\beta}}_{i} d v+\frac{\tau_{q}^{2}}{T_{0}} \int_{B} K_{i j} \overline{\overline{\beta_{j}}} \bar{\beta}_{i} d v \\
& +\frac{2 \tau_{q}}{T_{0}} \int_{0}^{t} \int_{B} \gamma_{i j} \overline{\bar{\beta}}_{j} \bar{\beta}_{i} d v d s+\frac{2}{T_{0}} \int_{0}^{t} \int_{0}^{s} \int_{B} \varkappa_{i j} \overline{\bar{\beta}}_{j} \bar{\beta}_{i} d v d z d s+\frac{\tau_{q}^{2}}{T_{0}} \int_{B} \gamma_{i j} \overline{\bar{\beta}}_{j} \bar{\beta}_{i} d v \\
& +\frac{\tau_{T} \tau_{q}^{2}}{T_{0}} \int_{0}^{t} \int_{B} k_{i j} \bar{\beta}_{j} \beta_{i} d v d s+\frac{2 \tau_{q}}{T_{0}} \int_{0}^{t} \int_{0}^{s} \int_{B} \kappa_{i j} \bar{\beta}_{j} \beta_{i} d v d z d s, \quad \forall t \geq 0 .
\end{aligned}
$$


At this point we need to invoke a conservation law, that we derive from Section 4, Equation (32), clearly here adapted to the case of assigned data all equal to zero. Namely:

$$
\begin{aligned}
& \frac{1}{2} \int_{0}^{t} \int_{0}^{s} \int_{B}\left[\rho \dot{u}_{i}^{*} \dot{u}_{i}^{*}+\rho \chi\left(\dot{\varphi}^{*}\right)^{2}+2 W^{*}+a\left(\dot{\alpha}^{*}\right)^{2}\right] d v d z d s+\frac{\tau_{q}^{2}}{4 T_{0}} \int_{B} K_{i j} \overline{\bar{\beta}}_{j} \overline{\bar{\beta}}_{i} d v \\
& +\frac{\tau_{q}}{2 T_{0}} \int_{0}^{t} \int_{B} K_{i j} \overline{\bar{\beta}}_{j} \overline{\bar{\beta}}_{i} d v d s+\frac{1}{2 T_{0}} \int_{0}^{t} \int_{0}^{s} \int_{B} K_{i j} \overline{\bar{\beta}}_{j} \overline{\bar{\beta}}_{i} d v d z d s+\frac{\tau_{q}^{2}}{4 T_{0}} \int_{0}^{t} \int_{B} \gamma_{i j} \bar{\beta}_{j} \bar{\beta}_{i} d v d s \\
& +\frac{1}{4 T_{0}} \int_{0}^{t} \int_{0}^{s} \int_{B}\left(2 \kappa_{i j}+3 \tau_{q} \varkappa_{i j}\right) \bar{\beta}_{j} \bar{\beta}_{i} d v d z d s+\frac{1}{T_{0}} \int_{0}^{t} \int_{0}^{s} \int_{0}^{z} \int_{B} \varkappa_{i j} \bar{\beta}_{j} \bar{\beta}_{i} d v d r d z d s \\
& +\frac{\tau_{T} \tau_{q}^{2}}{4 T_{0}} \int_{0}^{t} \int_{0}^{s} \int_{B} k_{i j} \beta_{j} \beta_{i} d v d z d s+\frac{\tau_{q}}{T_{0}} \int_{0}^{t} \int_{0}^{s} \int_{0}^{z} \int_{B} \kappa_{i j} \beta_{j} \beta_{i} d v d r d z d s=0 .
\end{aligned}
$$

In parallel, remembering the trivial assigned data, we start again from (13) $)_{1}$ multiplied by $u_{i}^{*}$ and from (13) $)_{2}$ multiplied by $\varphi^{*}$, we integrate over the region $B$ and use the divergence theorem, the Equations $(14)_{1}-(14)_{4},(15)_{1}$ as well as the definition of $W^{*}$ in order to get:

$$
\int_{B}\left[\rho u_{i}^{*} \ddot{u}_{i}^{*}+\rho \chi \varphi^{*} \ddot{\varphi}^{*}+2 W^{*}+a\left(\dot{\alpha}^{*}\right)^{2}\right] d v=\int_{B} \rho \eta^{*} \dot{\alpha}^{*} d v .
$$

We integrate now Equation (13) 3 , invoke the divergence theorem, use the Equation (14) 5 and the notation (15) 2 and integrate then the result twice with respect to the time variable. Through a series of laborious integrations by parts one is led to:

$$
\begin{aligned}
& \int_{0}^{t} \int_{0}^{s} \int_{B}\left[\rho u_{i}^{*} \ddot{u}_{i}^{*}+\rho \chi \varphi^{*} \ddot{\varphi}^{*}+2 W^{*}+a\left(\dot{\alpha}^{*}\right)^{2}\right] d v d z d s=-\frac{1}{T_{0}} \int_{0}^{t} \int_{B} K_{i j} \overline{\bar{\beta}}_{j} \overline{\bar{\beta}}_{i} d v d s \\
& -\frac{\tau_{q}}{T_{0}} \int_{B} K_{i j} \overline{\bar{\beta}}_{j} \overline{\bar{\beta}}_{i} d v-\frac{\tau_{q}^{2}}{2 T_{0}} \int_{B} K_{i j} \overline{\bar{\beta}}_{j} \bar{\beta}_{i} d v-\frac{\tau_{q}}{T_{0}} \int_{0}^{t} \int_{B} \gamma_{i j} \overline{\bar{\beta}}_{j} \bar{\beta}_{i} d v d s-\frac{1}{T_{0}} \int_{0}^{t} \int_{0}^{s} \int_{B} \varkappa_{i j} \overline{\bar{\beta}}_{j} \bar{\beta}_{i} d v d z d s \\
& -\frac{\tau_{q}^{2}}{2 T_{0}} \int_{B} \gamma_{i j} \overline{\bar{\beta}}_{j} \bar{\beta}_{i} d v-\frac{\tau_{T} \tau_{q}^{2}}{2 T_{0}} \int_{0}^{t} \int_{B} k_{i j} \bar{\beta}_{j} \beta_{i} d v d s-\frac{\tau_{q}}{T_{0}} \int_{0}^{t} \int_{0}^{s} \int_{B} \kappa_{i j} \bar{\beta}_{j} \beta_{i} d v d z d s \\
& -\frac{1}{2 T_{0}} \int_{0}^{t} \int_{0}^{s} \int_{B}\left(2 \tau_{T} k_{i j}-2 \tau_{q} \gamma_{i j}+\tau_{q}^{2} K_{i j}\right) \bar{\beta}_{j} \bar{\beta}_{i} d v d z d s+\frac{1}{T_{0}} \int_{0}^{t} \int_{0}^{s} \int_{B} K_{i j} \overline{\bar{\beta}}_{j} \overline{\bar{\beta}}_{i} d v d z d s \\
& +\frac{\tau_{q}^{2}}{2 T_{0}} \int_{B} K_{i j} \overline{\bar{\beta}}_{j} \overline{\bar{\beta}}_{i} d v+\frac{\tau_{T} \tau_{q}^{2}}{2 T_{0}} \int_{0}^{t} \int_{0}^{s} \int_{B} k_{i j} \beta_{j} \beta_{i} d v d z d s \\
& +\frac{\tau_{q}}{T_{0}} \int_{0}^{t} \int_{B} K_{i j} \overline{\bar{\beta}}_{j} \overline{\bar{\beta}}_{i} d v d s+\frac{\tau_{q}^{2}}{2 T_{0}} \int_{0}^{t} \int_{B} \gamma_{i j} \bar{\beta}_{j} \bar{\beta}_{i} d v d s .
\end{aligned}
$$


Such a relation has to be included in the conservation law (48), properly manipulated to receive it. Therefore we come to the following relation:

$$
\begin{aligned}
& \int_{0}^{t} \int_{0}^{s} \int_{B}\left[\rho \dot{u}_{i}^{*} \dot{u}_{i}^{*}+\rho \chi\left(\dot{\varphi}^{*}\right)^{2}\right] d v d z d s+\frac{\tau_{q}^{2}}{T_{0}} \int_{B} K_{i j} \overline{\bar{\beta}}_{j} \overline{\bar{\beta}}_{i} d v+\frac{2 \tau_{q}}{T_{0}} \int_{0}^{t} \int_{B} K_{i j} \overline{\bar{\beta}}_{j} \overline{\bar{\beta}}_{i} d v d s \\
& +\frac{2}{T_{0}} \int_{0}^{t} \int_{0}^{s} \int_{B} K_{i j} \overline{\bar{\beta}}_{j} \overline{\bar{\beta}}_{i} d v d z d s+\frac{\tau_{q}^{2}}{T_{0}} \int_{0}^{t} \int_{B} \gamma_{i j} \bar{\beta}_{j} \bar{\beta}_{i} d v d s+\frac{2 \tau_{q}}{T_{0}} \int_{0}^{t} \int_{0}^{s} \int_{B} \varkappa_{i j} \bar{\beta}_{j} \bar{\beta}_{i} d v d z d s \\
& +\frac{2}{T_{0}} \int_{0}^{t} \int_{0}^{s} \int_{0}^{z} \int_{B} \varkappa_{i j} \bar{\beta}_{j} \bar{\beta}_{i} d v d r d z d s+\frac{\tau_{T} \tau_{q}^{2}}{T_{0}} \int_{0}^{t} \int_{0}^{s} \int_{B} k_{i j} \beta_{j} \beta_{i} d v d z d s \\
& +\frac{2 \tau_{q}}{T_{0}} \int_{0}^{t} \int_{0}^{s} \int_{0}^{z} \int_{B} \kappa_{i j} \beta_{j} \beta_{i} d v d r d z d s \\
& =\int_{0}^{t} \int_{0}^{s} \int_{B}\left(\rho u_{i}^{*} \ddot{u}_{i}^{*}+\rho \chi \varphi^{*} \ddot{\varphi}^{*}\right) d v d z d s+\frac{1}{T_{0}} \int_{0}^{t} \int_{B} K_{i j} \overline{\bar{\beta}}_{j} \overline{\bar{\beta}}_{i} d v d s+\frac{\tau_{q}}{T_{0}} \int_{B} K_{i j} \overline{\bar{\beta}}_{j} \overline{\bar{\beta}}_{i} d v \\
& +\frac{\tau_{q}^{2}}{2 T_{0}} \int_{B} K_{i j} \overline{\bar{\beta}}_{j} \bar{\beta}_{i} d v+\frac{\tau_{q}}{T_{0}} \int_{0}^{t} \int_{B} \gamma_{i j} \overline{\bar{\beta}}_{j} \bar{\beta}_{i} d v d s+\frac{1}{T_{0}} \int_{0}^{t} \int_{0}^{s} \int_{B} \varkappa_{i j} \overline{\bar{\beta}}_{j} \bar{\beta}_{i} d v d z d s \\
& +\frac{\tau_{q}^{2}}{2 T_{0}} \int_{B} \gamma_{i j} \overline{\bar{\beta}}_{j} \bar{\beta}_{i} d v+\frac{\tau_{T} \tau_{q}^{2}}{2 T_{0}} \int_{0}^{t} \int_{B} k_{i j} \bar{\beta}_{j} \beta_{i} d v d s+\frac{\tau_{q}}{T_{0}} \int_{0}^{t} \int_{0}^{s} \int_{B} \kappa_{i j} \bar{\beta}_{j} \beta_{i} d v d z d s .
\end{aligned}
$$

The Equation (51) has to be multiplied by two; then, the term in which we integrate $2\left(\rho u_{i}^{*} \ddot{u}_{i}^{*}+\rho \chi \varphi^{*} \ddot{\varphi}^{*}\right)$ has to be isolated and replaced into the second derivative of the functional $\mathscr{F}\left(\mathscr{S}^{*}\right)(t)$, i.e., Equation (47). This operation returns:

$$
\begin{aligned}
& \frac{d^{2} \mathscr{F}\left(\mathscr{S}^{*}\right)}{d t^{2}}(t)=4\left[\int_{0}^{t} \int_{0}^{s} \int_{B}\left[\rho \dot{u}_{i}^{*} \dot{u}_{i}^{*}+\rho \chi\left(\dot{\varphi}^{*}\right)^{2}\right] d v d z d s+\frac{\tau_{q}^{2}}{2 T_{0}} \int_{B} K_{i j} \overline{\bar{\beta}}_{j} \overline{\bar{\beta}}_{i} d v\right. \\
& +\frac{\tau_{q}}{T_{0}} \int_{0}^{t} \int_{B} K_{i j} \overline{\bar{\beta}}_{j} \overline{\bar{\beta}}_{i} d v d s+\frac{1}{T_{0}} \int_{0}^{t} \int_{0}^{s} \int_{B} K_{i j} \overline{\bar{\beta}}_{j} \overline{\bar{\beta}}_{i} d v d z d s+\frac{\tau_{q}^{2}}{2 T_{0}} \int_{0}^{t} \int_{B} \gamma_{i j} \bar{\beta}_{j} \bar{\beta}_{i} d v d s \\
& +\frac{\tau_{q}}{T_{0}} \int_{0}^{t} \int_{0}^{s} \int_{B} \varkappa_{i j} \bar{\beta}_{j} \bar{\beta}_{i} d v d z d s+\frac{1}{T_{0}} \int_{0}^{t} \int_{0}^{s} \int_{0}^{z} \int_{B} \varkappa_{i j} \bar{\beta}_{j} \bar{\beta}_{i} d v d r d z d s \\
& \left.+\frac{\tau_{T} \tau_{q}^{2}}{2 T_{0}} \int_{0}^{t} \int_{0}^{s} \int_{B} k_{i j} \beta_{j} \beta_{i} d v d z d s+\frac{\tau_{q}}{T_{0}} \int_{0}^{t} \int_{0}^{s} \int_{0}^{z} \int_{B} \kappa_{i j} \beta_{j} \beta_{i} d v d r d z d s\right] .
\end{aligned}
$$

In conclusion, we take as a reference the Equation (45) for the functional $\mathscr{F}\left(\mathscr{S}^{*}\right)(t)$, the Equation (46) for its first derivative in time and the Equation (52) for its second derivative. Invoking the Cauchy-Schwarz inequality, we can say that:

$$
\mathscr{F}\left(\mathscr{S}^{*}\right)(t) \frac{d^{2} \mathscr{F}\left(\mathscr{S}^{*}\right)}{d t^{2}}(t) \geq\left[\frac{d \mathscr{F}\left(\mathscr{S}^{*}\right)}{d t}(t)\right]^{2}
$$

and so the desired logarithmic convexity condition in $t$ is achieved. In view of the trivial initial data selected here, the functional $\mathscr{F}\left(\mathscr{S}^{*}\right)(t)$ is therefore identically null, a condition thanks to which the uniqueness of the solution is confirmed (see again $[36,38]$ ).

\section{Conclusions}

With this work we have contributed to the definition of the well-posedness issue for a time-differential three-phase lag linear thermoelastic model involving a porous elastic matrix. In particular, the presence of voids into the elastic skeleton was taken into consideration relying on the Cowin-Nunziato theory [28-31], while the thermodynamic behavior was supposed to undergo a constitutive equation with three delay times, as suggested by Roy Choudhuri [2]. The proposed initial 
boundary value problem was suitably modified thanks to the linearity of the model: two uniqueness results have been achieved, considering both the Lagrange identity method and the logarithmic convexity method, in addition to a continuous dependence theorem. We emphasize that such results requested the assumption of mild restrictions upon the constitutive coefficients and the (positive) delay times, which at most coincided with the conditions of thermodynamic compatibility (TC) of the model derived in [35].

Author Contributions: M.C. conceptualization, formal analysis and writing-original draft preparation. V.Z. methodology, writing-review and editing. Both authors declare that they have contributed equally to the realization of this work. Both authors have read and agreed to the published version of the manuscript.

Funding: This research received no external funding.

Acknowledgments: V. Zampoli acknowledges the GNFM (INdAM Gruppo Nazionale per la Fisica Matematica) for the support to his research activity, as well as for the research projects Progetti Giovani 2018 and 2019. The authors would like to thank both the reviewers for their careful analysis of the manuscript and their comments.

Conflicts of Interest: The authors declare no conflict of interest.

\section{Abbreviations}

The following abbreviations are used in this manuscript:

TC Thermodynamic compatibility

LHS Left hand side

RHS Right hand side

\section{References}

1. Tzou, D.Y. Macro- to Microscale Heat Transfer: The Lagging Behavior, 2nd ed.; John Wiley and Sons: Chichester, UK, 2014; ISBN 9781118818220. [CrossRef]

2. Roy Choudhuri, S.K. On a thermoelastic three-phase-lag model. J. Therm. Stresses 2007, 30, $231-238$. [CrossRef]

3. Quintanilla, R. A well posed problem for the dual-phase-lag heat conduction. J. Therm. Stresses 2008, 31, 260-269. [CrossRef]

4. Quintanilla, R.; Racke, R. A note on stability in three-phase-lag heat conduction. Int. J. Heat Mass Transfer 2008, 51, 24-29. [CrossRef]

5. Quintanilla, R. A well-posed problem for the three-dual-phase-lag heat conduction. J. Therm. Stresses 2009, 32, 1270-1278. [CrossRef]

6. Kumar, R.; Mukhopadhyay, S. Effects of three phase lags on generalized thermoelasticity for an infinite medium with a cylindrical cavity. J. Therm. Stresses 2009, 32, 1149-1165. [CrossRef]

7. Mukhopadhyay, S.; Kothari, S.; Kumar, R. On the representation of solutions for the theory of generalized thermoelasticity with three phase-lags. Acta Mech. 2010, 214, 305-314. [CrossRef]

8. Zampoli, V.; Landi, A. A domain of influence result about the time differential three-phase-lag thermoelastic model. J. Therm. Stresses 2017, 40, 108-120. [CrossRef]

9. Biswas, S.; Mukhopadhyay, B.; Shaw, S. Rayleigh surface wave propagation in orthotropic thermoelastic solids under three-phase-lag model. J. Therm. Stresses 2017, 40, 403-419. [CrossRef]

10. Magaña, A.; Quintanilla, R. On the existence and uniqueness in phase-lag thermoelasticity. Meccanica 2018, 53, 125-134. [CrossRef]

11. Magaña, A.; Miranville, A.; Quintanilla, R. On the stability in phase-lag heat conduction with two temperatures. J. Evol. Equ. 2018, 18, 1697-1712. [CrossRef]

12. Zampoli, V. Uniqueness theorems about high-order time differential thermoelastic models. Ric. di Mat. 2018, 67, 929-950. [CrossRef]

13. Zampoli, V. Some continuous dependence results about high-order time differential thermoelastic models.

J. Therm. Stresses 2018, 41, 827-846. [CrossRef]

14. Chiriţă, S.; Zampoli, V. Spatial behavior of the dual-phase-lag deformable conductors. J. Therm. Stresses 2018, 41, 1276-1296. [CrossRef] 
15. Zampoli, V. On the increase in signal depth due to high-order effects in micro- and nanosized deformable conductors. Math. Prob. Eng. 2019, 2019, 2629012. [CrossRef]

16. Mondal, S.; Sur, A.; Kanoria, M. A memory response in the vibration of a microscale beam induced by laser pulse. J. Therm. Stresses 2019, 42, 1415-1431. [CrossRef]

17. Bazarra, N.; Campo, M.; Fernández, J.R.; Quintanilla, R. Numerical analysis of a thermoelastic problem with dual-phase-lag heat conduction. Appl. Numer. Math. 2019, 140, 76-90. [CrossRef]

18. Bazarra, N.; Copetti, M.I.M.; Fernández, J.R.; Quintanilla, R. Numerical analysis of some dual-phase-lag models. Comput. Math. Appl. 2019, 77, 407-426. [CrossRef]

19. Bazarra, N.; Bochicchio, I.; Fernández, J.R.; Naso, M.G. Analysis of a contact problem involving an elastic body with dual-phase-lag. Appl. Math. Optim. 2019. [CrossRef]

20. Abo-Dahab, S.M.; Biswas, S. P, T, and SV wave propagation at the interface between solid-liquid media with magnetic field and initial stress in the context of three-phase-lag model. Mech. Adv. Mater. Struct. 2020, 27, 165-175. [CrossRef]

21. Kumar, H.; Mukhopadhyay, S. Thermoelastic damping analysis for size-dependent microplate resonators utilizing the modified couple stress theory and the three-phase-lag heat conduction model. Int. J. Heat Mass Transfer 2020, 148. [CrossRef]

22. Sur, A. Wave propagation analysis of porous asphalts on account of memory responses. Mech. Based Des. Struct. Mach. 2020. [CrossRef]

23. Green, A.E.; Naghdi, P.M. On undamped heat waves in an elastic solid. J. Therm. Stresses 1992, 15, $253-264$. [CrossRef]

24. Green, A.E.; Naghdi, P.M. Thermoelasticity without energy dissipation. J. Elast. 1993, 31, 189-208. [CrossRef]

25. Green, A.E.; Naghdi, P.M. A unified procedure for construction of theories of deformable media. I. Classical continuum physics, II. Generalized continua, III. Mixtures of interacting continua. Proc. R. Soc. Lond. Ser. A 1995, 448, 335-356 (doi:10.1098/rspa.1995.0020), 357-377 (doi:10.1098/rspa.1995.0021), 379-388 (doi:10.1098/rspa.1995.0022).

26. Zampoli, V.; Amendola, A. Uniqueness, continuous dependence, and spatial behavior of the solution in linear porous thermoelasticity with two relaxation times. J. Therm. Stresses 2019, 42, 1582-1602. [CrossRef]

27. Carini, M.; Zampoli, V. On the influence of voids on the depth of an external signal in thermoelasticity with two relaxation times. Acta Mech. 2020, 231, 363-371. [CrossRef]

28. Cowin, S.C.; Nunziato, J.W. Linear elastic materials with voids. J. Elast. 1983, 13, 125-147. [CrossRef]

29. Ieşan, D. Some theorems in the theory of elastic materials with voids. J. Elast. 1985, 15, 215-224. [CrossRef]

30. Ieşan, D. A theory of thermoelastic materials with voids. Acta Mech. 1986, 60, 67-89. [CrossRef]

31. Ieşan, D. Thermoelastic Models of Continua, Solid Mechanics and Its Applications; Kluwer Academic Publishers: Berlin, Germany, 2004; Volume 118; ISBN 978-90-481-6634-3. [CrossRef]

32. Almutairi, F.; Khaled, S.M.; Ebaid, A. MHD flow of nanofluid with homogeneous-heterogeneous reactions in a porous medium under the influence of second-order velocity slip. Mathematics 2019, 7, 220. [CrossRef]

33. Badruddin, I.A. Numerical analysis of thermal non-equilibrium in porous medium subjected to internal heating. Mathematics 2019, 7, 1085. [CrossRef]

34. D'Apice, C.; Chiriţă, S.; Zampoli, V. On the well-posedness of the time-differential three-phase-lag thermoelasticity model. Arch. Mech. 2016, 68, 371-393.

35. Chiriţă, S.; D'Apice, C.; Zampoli, V. The time differential three-phase-lag heat conduction model: Thermodynamic compatibility and continuous dependence. Int. J. Heat Mass Transfer 2016, 102, 226-232. [CrossRef]

36. Chiriţă, S. Modeling triple porosity under local thermal non-equilibrium. J. Therm. Stresses 2020, 43, $210-224$. [CrossRef]

37. Knops, R.J.; Payne, L.E. Uniqueness in classical elastodynamics. Arch. Ration. Mech. Anal. 1968, $27,349-355$. [CrossRef]

38. Ames, W.; Straughan, B. Non-Standard and Improperly Posed Problems. Mathematics in Science and Engineering; Academic Press: San Diego, CA, USA, 1997; Volume 194; ISBN 9780120567454.

(C) 2020 by the authors. Licensee MDPI, Basel, Switzerland. This article is an open access article distributed under the terms and conditions of the Creative Commons Attribution (CC BY) license (http:/ / creativecommons.org/licenses/by/4.0/). 